\title{
CC-115, a Dual Mammalian Target of Rapamycin/ DNA-Dependent Protein Kinase Inhibitor in Clinical Trial, Is a Substrate of ATP-Binding Cassette G2, a Risk Factor for CC-115 Resistance ${ }^{\mathbb{S}}$
}

\author{
Jenny Beebe and (D) Jian-Ting Zhang \\ Department of Pharmacology and Toxicology (J.B., J.-T.Z.) and IU Simon Cancer Center (J.-T.Z.), Indiana University School of \\ Medicine, Indianapolis, Indiana; and Department of Cancer Biology, University of Toledo College of Medicine and Life Sciences, \\ Toledo, Ohio (J.-T.Z.)
}

Received March 27, 2019; accepted August 16, 2019

\section{ABSTRACT}

CC-115, a triazole-containing compound, is a dual mammalian target of rapamycin (mTOR)/DNA-dependent protein kinase (DNA-PK) inhibitor currently in clinical trials. To develop this compound further, we investigated factors that may affect cellular response to CC-115. Previously, fatty acid synthase (FASN) was shown to upregulate DNA-PK activity and contribute to drug resistance; therefore, we hypothesized that FASN may affect cellular response to CC-115. Instead, however, we showed that $\mathrm{CC}-115$ is a substrate of ATP-binding cassette G2 (ABCG2), a member of the ATP-binding cassette transporter superfamily, and that expression of ABCG2, not FASN, affects the potency of $\mathrm{CC}-115$. ABCG2 overexpression significantly increases resistance to CC-115. Inhibiting ABCG2 function, using small-molecule inhibitors, sensitizes cancer cells to $C C$ 115. We also found that CC-115 may be a substrate of $A B C B 1$, another known $\mathrm{ABC}$ protein that contributes to drug resistance. These findings suggest that expression of $A B C$ transporters, including $A B C B 1$ and $A B C G 2$, may affect the outcome in clinical trials testing $C C-115$. Additionally, the data indicate that $A B C$ transporters may be used as markers for future precision use of CC-115

\section{SIGNIFICANCE STATEMENT}

In this article, we report our findings on the potential mechanism of resistance to CC-115, a dual inhibitor of mTOR and DNA-PK currently in clinical trials. We show that CC-115 is a substrate of $A B C G 2$ and can be recognized by $A B C B 1$, which contributes to $C C-115$ resistance. These findings provide novel information and potential guidance on future clinical testing of CC-115.

\section{Introduction}

The phosphoinositide 3-kinase (PI3) K-related kinase (PIKK) family of serine/threonine kinases includes ataxia-telangiectasia mutated, ataxia-telangiectasia mutated related, human suppressor of morphogenesis in genitalia-1, DNA-PK, and mTOR (Lovejoy and Cortez, 2009). These protein kinases regulate cellular response to DNA damage and to nutrients, controlling cell growth (Lovejoy and Cortez, 2009). In particular, DNA-PK plays an important role in nonhomologous end joining repair of DNA double-strand breaks (Hammel et al., 2010), whereas mTOR functions in regulating protein synthesis, cell proliferation, and survival (Hung et al., 2012). Both DNA-PK and mTOR have been considered as targets for drug discovery.

CC-115 $\left(\mathrm{C}_{16} \mathrm{H}_{16} \mathrm{~N}_{8} \mathrm{O}\right)$, a triazole-containing compound, is a dual mTOR/DNA-PK inhibitor currently in clinical trials

https://doi.org/10.1124/jpet.119.258392.

S This article has supplemental material available at jpet.aspetjournals.org.
(Mortensen et al., 2015). Early studies showed that CC-115 was selective to mTOR and DNA-PK over other closely related PIKK family members (Tsuji et al., 2017). Several ongoing clinical trials are testing CC-115 in an array of different cancer types, including phase 1 trials in squamous cell carcinoma of head and neck, Ewing's osteosarcoma, chronic lymphocytic leukemia (CLL), and prostate cancer (Munster et al., 2016). There is also a phase 2 trial in glioblastoma. Early findings on CC-115 in CLL patients showed that seven of eight patients had a decrease in lymphadenopathy, with one partial response and three partial responses with lymphocytosis (Thijssen et al., 2016). Although these early results in CLL show promise for further development of CC-115, there is an observed variability in sensitivity. In fact, one of the eight CLL patients showed no decrease in lymphadenopathy and may be resistant to CC-115. Clearly, resistance to CC115 will likely be observed, and there is a need to identify the mechanism of CC-115 resistance and to overcome this resistance for better development and clinical use of CC-115.

ABBREVIATIONS: ABC, ATP-binding cassette; CLL, chronic lymphocytic leukemia; DMEM, Dulbecco's modified Eagle's medium; DNA-PK, DNAdependent protein kinase; FASN, fatty acid synthase; FBS, fetal bovine serum; FTC, fumitremorgin C; HEK293, human embryonic kidney cell line 293; mTOR, mammalian target of rapamycin; MTT, 3-(4,5-Dimethylthiazol-2-yl)-2,5-diphenyltetrazolium bromide; PBS, phosphate-buffered saline; P13K, phosphoinositide 3-kinase; PIKK, PI3K-related kinase. 
There are many known molecular mechanisms of drug resistance. ATP-binding cassette $(\mathrm{ABC})$ transporters, such as ABCB1, $\mathrm{ABCC} 1$, and ABCG2, are particularly well known as contributing to multidrug resistance by actively transporting drug substrates out of cells using ATP (Schinkel and Jonker, 2003). Fatty acid synthase (FASN) is known to contribute to resistance of DNA-damaging drugs by increasing nonhomologous end joining repair of DNA damages via facilitating recruitment of Ku proteins and increasing DNAPK activity (Wu et al., 2016a). Thus, it is possible that FASN may contribute to CC-115 resistance by increasing DNA-PK activity. In this study, we tested this hypothesis; however, we found that ABCG2, not FASN, contributes to CC-115 resistance by reducing CC- 115 accumulation. Inhibiting ABCG2 using small-molecule inhibitors was able to reverse CC-115 resistance. These findings suggest that $\mathrm{CC}-115$ is a substrate of ABCG2, and ABCG2 expression likely will cause resistance to CC-115.

\section{Materials and Methods}

Materials. Antibodies against ABCG2 (no. MAB4146), DNA-PK (no. PC127), and actin (no. JLA-20) were from Millipore (Danvers, MA), Calbiochem (San Diego, CA), and Sigma (Saint Louis, MO), respectively. Antibodies against mTOR (no. 2983, 1:500), p-S6 (no. 2215, 1:1000), S6 (no. 2317, 1:200), p-S6K (no. 9234, 1:1000), S6K (no. 2708, 1:1000), p-AKT (no. 4051, 1:1000) were all from Cell Signaling (Danvers, MA). Antibody against DNA-PKcs (no. PIMA513238, 1: 1000) was from Invitrogen (Carlsbad, CA). S6K and p-S6K antibodies can also detect p85. Actin (no. JLA-20, 1:3000), anti-mouse secondary (no. A2554, 1:3000), and anti-rabbit secondary (no. A0545, 1:3000) antibodies were from Sigma. CC-115 (no. S7891) and NU7441 (no. S2638) were obtained from Selleckchem (Houston, TX), and fumitremorgin C (FTC) (\#344847) was obtained from Millipore (Burlington, MA). PZ-39C8 was obtained from SPECS (www.specs.net)as previously reported (Peng et al., 2009). G418 (no. G5005) was purchased from TEKnova (Hollister, CA). Doxorubicin (no. D1515) and thiazolyl blue tetrazolium bromide (no. M5655) were from Sigma. Protein concentration assay kit and polyvinylidene difluoride membranes were purchased from Bio-Rad Laboratories (Hercules, CA). All other chemicals were of molecular biology grade from Fisher Scientific or Sigma.

Cell Lines. MCF7 and M3K cells were cultured in Dulbecco's modified Eagle's medium (DMEM) (no. 10-013-CV) (Corning, Manassas, VA) containing 10\% fetal bovine serum (FBS) (no. A3160601) (Life Technologies, Grand Island, NY) with supplementation of $5 \mu \mathrm{M}$ doxorubicin for M3K cells as previously described (Liu et al., 2006).
MCF7 cells with stable expression of ectopic FASN (MCF7/FASN) and M3K cells with stable knockdown of FASN (M3K/ShFASN), along with their respective control cells (MCF7/Vec and M3K/Scr), were established previously and cultured in DMEM containing 10\% FBS and $600 \mu \mathrm{g} / \mathrm{ml} \mathrm{G418}$ as previously described (Liu et al., 2008). Human embryonic kidney cell line 293 (HEK293)/ABCG2-venus and HEK293/ Vec-venus were also established in a previous study and cultured in DMEM containing 10\% FBS with $600 \mu \mathrm{g} / \mathrm{ml}$ G418 as previously described (W. Zhang et al., submitted manuscript). MCF7 cells with stable expression of ectopic ABCB1 (BC19) were established previously (Horton et al., 1998) and cultured similarly in DMEM containing $10 \%$ FBS with $0.1 \mu \mathrm{M}$ Adriamycin.

To establish MCF7/ABCG2 cells with stable expression of ectopic ABCG2, MCF7 cells were transfected with pcDNA3.1 (+) harboring ABCG2 cDNA or with vector alone, followed by selection with 600 $\mu \mathrm{g} / \mathrm{ml} \mathrm{G} 418$, cloned, and propagated under the same conditions.

Survival Assays. We plated 500 to 4000 cells/well in a 96 -well plate and cultured overnight, followed by treatment with increasing concentrations of CC-115 in the absence or presence of doxorobucin at its $\mathrm{IC}_{20}$ concentration for 3 days, and cell survival was measured using methylene-blue staining (Oliver et al., 1989) or 3-(4,5-Dimethylthiazol-2-yl)-2,5-diphenyltetrazolium bromide for (MTT) assay. For methylene blue assay, cells were first fixed with methanol for 30 minutes and stained with $1 \%(\mathrm{w} / \mathrm{v})$ methylene-blue in $10 \mathrm{mM}$ borate buffer, $\mathrm{pH} 8.5$, for 30 minutes. Excess dye was removed by aspiration, and plates were washed three times with $\mathrm{dd}_{2} \mathrm{O}$. The stains were then released using a 1:1 mixture of $100 \%$ ethanol and $0.1 \mathrm{M} \mathrm{HCl}$, followed by determination of O.D.650nm. For MTT assay, cells were treated with $5 \mathrm{mg} / \mathrm{ml}$ thiazolyl blue tetrazolium bromide in phosphate-buffered saline (PBS) for 4 hours at $37^{\circ} \mathrm{C}$, and cells were collected by centrifugation. Finally, dimethylsulfoxide was added to solubilize cellassociated dye, and $\mathrm{OD}_{570 \mathrm{~nm}}$ was determined.

Cell Lysate Preparation and Western Blot. Cells were treated with the given concentration of CC-115 for 2 hours, and cell lysates were prepared as previously described (Liu et al., 2006) and separated by SDS-PAGE, followed by transfer onto polyvinylidene difluoride membranes. Western blot analysis was also performed exactly as we previously described (Xu et al., 2004, 2007). To test the effect of ABCG2 inhibition on CC-115 inhibition of mTOR, $1 \mu \mathrm{M}$ C8 was added at the same time as CC-115 and incubated for 2 hours before cell lysates were prepared.

CC-115 Accumulation Assay. Cells were harvested, washed twice with warm PBS, and incubated with or without ABCG2 inhibitors for 15 minutes at $37^{\circ} \mathrm{C}$. Then, $10 \mu \mathrm{M}$ CC- 115 or dimethylsulfoxide control was added to the cells, followed by incubation at $37^{\circ} \mathrm{C}$ for 30 minutes with shaking every 10 minutes. Cells were then washed with PBS twice and subjected to analysis using flow cytometry with an excitation at $405 \mathrm{~nm}$ and emission at $421 \mathrm{~nm}$.
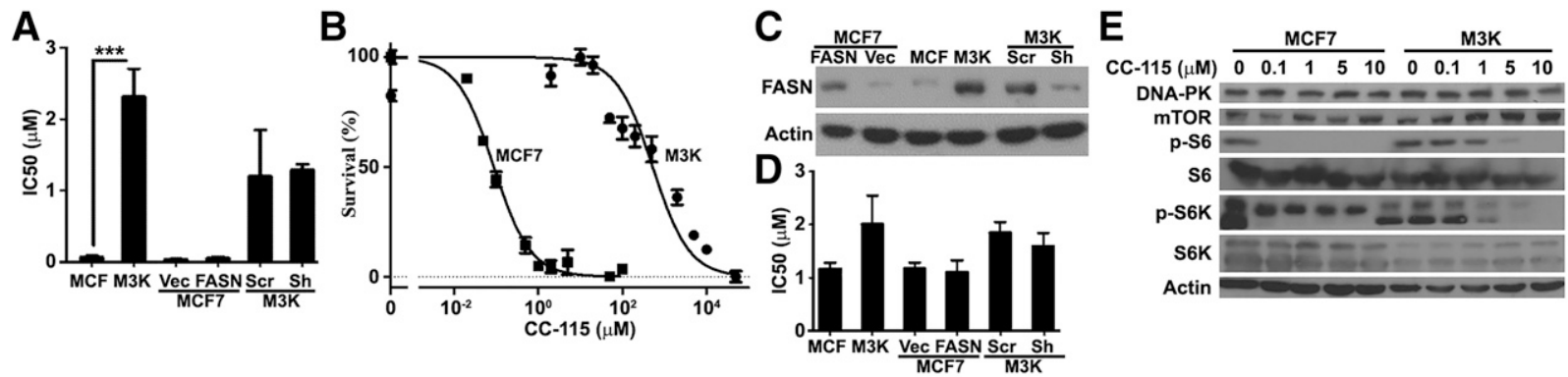

Fig. 1. FASN does not contribute to DNA-PK inhibitor resistance. (A) $\mathrm{IC}_{50}$ of CC-115 in different cell lines as determined from dose-dependent survival curves using methylene blue assay. (B) Dose-dependent survival curve of MCF7 and M3K cells in the presence of CC-115. (C) FASN expression in MCF7/Vec, MCF7/FASN, MCF7, M3K, M3K/Scr, and, M3K/shFASN cell lines. (D) IC 50 of the DNA-PK inhibitor Nu7441 as determined from dose-dependent survival curve using methylene-blue assay. (E) CC-115 inhibition of mTOR, monitored by phosphorylation of S6 and S6K (lower band) as determined using Western blot analyses. Note that p85 was also detected by S6K and pS6K antibodies. Statistical analysis using ANOVA comparing all groups was used in (A and E), followed by Tukey post hoc test $(n=3 ; * P<0.05=, * * P<0.01$, $* * * P<0.001)$. DNA-PK, DNA-PKcs. 


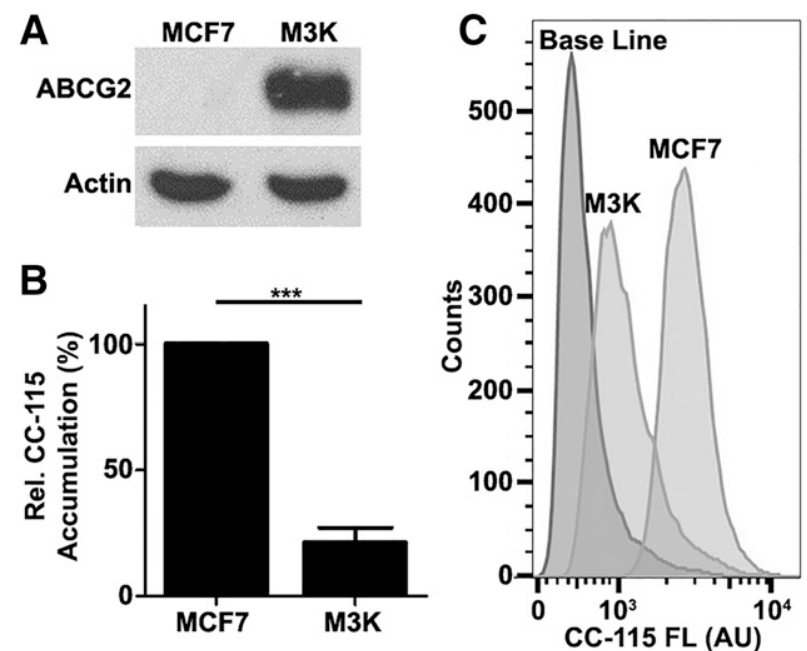

Fig. 2. Identification of ABCG2 as a resistance factor for CC-115. (A) Expression of ABCG2 in MCF7 and M3K cells as determined using Western blot analysis. (B and C) CC-115 accumulation in MCF7 and M3K cells determined using fluorescence-activated cell sorter (FACS) analysis. The $t$ test was used to compare CC-115 accumulation (B) in different cells $(n=3$; $* P<0.05=$, $* * P<0.01$, $* * * P<0.001)$.

Statistical Analysis. Statistical analysis for all experiments was run using Prism Graphpad (San Diego, CA). Results are presented as mean \pm standard deviation. IC $_{50}$ values were calculated from three biologic replicates, each run in triplicate. Western blots were run on four different sample preps, and accumulation assays were run in triplicate. Two-tailed $t$ test was used for comparing two means and ANOVA using the Tukey post hoc test for comparing a group of mean values.

\section{Results}

FASN Does Not Contribute to Cellular Resistance to DNA-PK Inhibitors. To investigate the potential mechanisms of CC-115 resistance, we first took advantage of the MCF7/AdVp3000 (M3K) cell line, which was selected for Adriamycin resistance. M3K cells harbor multiple mechanisms of drug resistance, including overexpression of FASN (Liu et al., 2008), and were tested for their response to CC-115 in comparison with the parental MCF7 cells. As shown in Fig. 1, A and B, M3K cells are much more resistant than MCF7 cells to CC-115 ( $\mathrm{IC}_{50}$ of $\sim 2.2$ and $\sim 0.05 \mu \mathrm{M}$, respectively).

Previously, it has been shown that CC-115 inhibits DNA-PK (Tsuji et al., 2017) and that FASN increases DNA-PK activity in M3K cells (Wu et al., 2016b). Thus, it is possible that FASN overexpression in M3K cells contribute to CC-115 resistance. To test this possibility, we took advantage of MCF7 cells with stable overexpression of ectopic FASN (MCF7/FASN) and M3K cells with stable FASN knockdown (M3K/ShFASN) (see Fig. 1C) and tested their response to CC-115 compared with their respective control cells (MCF7/Vec and M3K/Scr). As shown in Fig. 1A and Supplemental Fig. S1, ectopic FASN overexpression or FASN knockdown did not influence cellular response to CC-115. Thus, FASN may not contribute to CC-115 resistance via DNA-PK.

To ensure that FASN expression does not contribute to resistance to DNA-PK inhibition, we tested a DNA-PK selective inhibitor, NU7441. As shown in Fig. 1D and Supplemental Fig. S2, alteration of FASN expression did not change cellular response to NU7441, although M3K cells are slightly more resistant to NU7441 than the parental MCF7 cells. DNA-PK activation occurs in response to DNA damage,

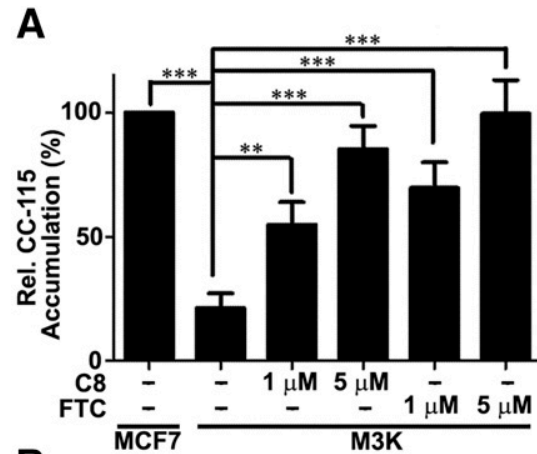

B

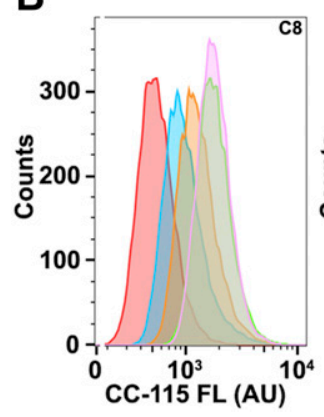

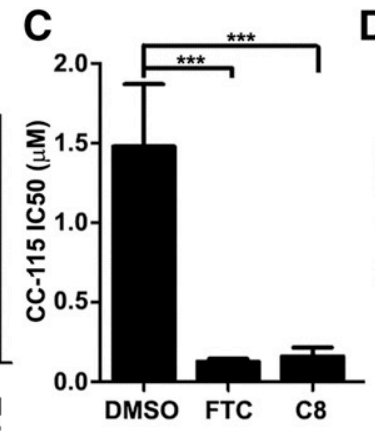

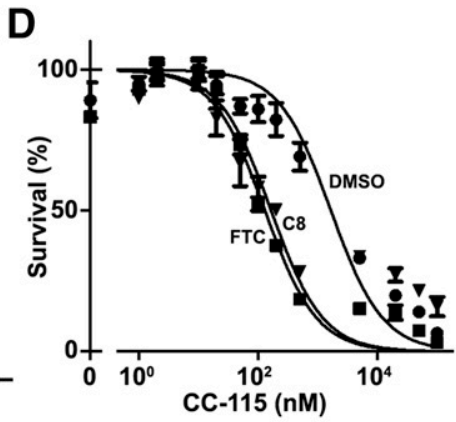

E

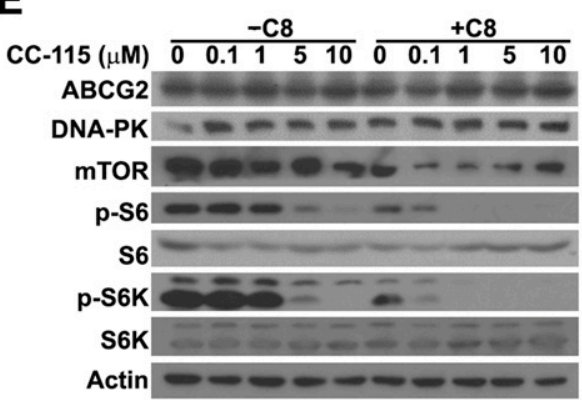

Fig. 3. Inhibition of ABCG2 and reversal of CC-115 resistance. (A and B) CC-115 accumulation in M3K cells in the presence of ABCG2 inhibitors C8 and FTC as determined using fluorescence-activated cell sorter (FACS) analysis. (C and D) CC-115 IC I0 $_{50}$ in and representative dose-dependent survival curves of M3K cells in the absence or presence of $1 \mu \mathrm{M}$ ABCG2 inhibitor C8 or FTC as determined using methylene-blue assay. (E) CC-115 inhibition of mTOR activation in the absence or presence of $1 \mu \mathrm{M}$ ABCG2 inhibitor C8 as determined by monitoring phosphorylation of S6 and S6K using Western blot analysis. Note that $\mathrm{p} 85$ was also detected by S6K and pS6K antibodies. ANOVA statistical analysis was used to compare CC-115 accumulation (A) and $\mathrm{IC}_{50}(\mathrm{C})$ in different cells $\left(n=3\right.$ to $4 ; * P<0.05, * * P<0.01$, $\left.{ }^{* * *} P<0.001\right)$. DNA-PK, DNA-PKcs. 

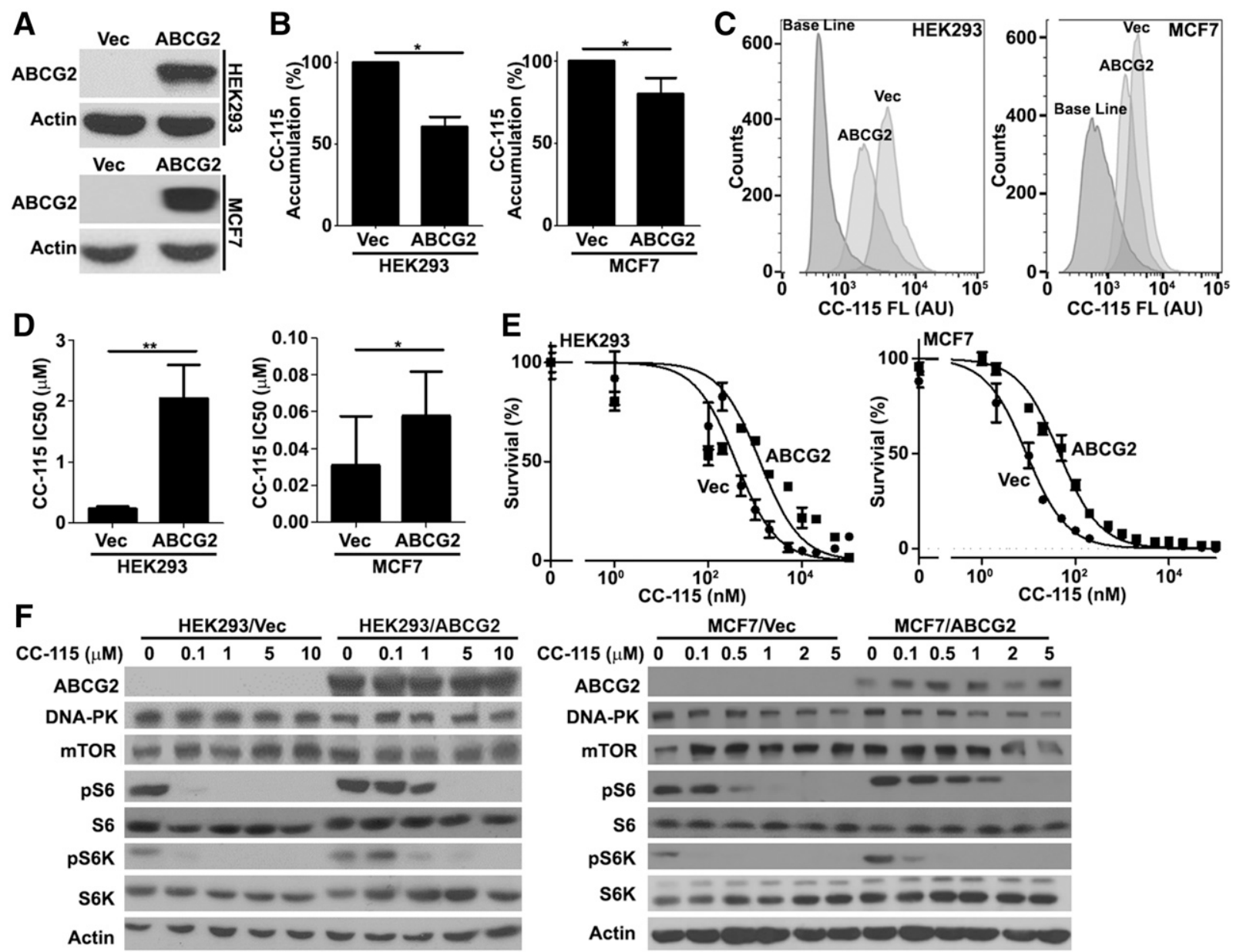

Fig. 4. Effect of ABCG2 overexpression on CC-115 accumulation and resistance. (A) Western blot analysis of ABCG2 expression in HEK293/Vec versus HEK293/ABCG2-venus and MCF7/Vec versus MCF7/FASN cells. (B and C) CC-115 accumulation in HEK293/Vec versus HEK293/ABCG2-venus and

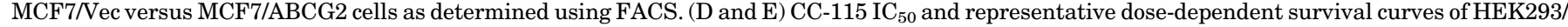
Vec versus HEK293/ABCG2-venus and MCF7/Vec versus MCF7/ABCG2 cells as determined using methylene-blue assay for HEK293 and MTT assay for MCF7 cells. (F) CC-115 inhibition of mTOR activation in HEK293/Vec versus HEK293/ABCG2-venus and MCF7/Vec versus MCF7/ABCG2 cells as determined by monitoring phosphorylation of S6 and S6K using Western blot analysis. Note that p85 was also detected by S6K and pS6K antibodies. The $t$ test was used to compare CC-115 accumulation (B) and $\mathrm{IC}_{50}$ (D) $\left(n=3\right.$ to 4 ; ${ }^{*} P<0.05$, $* * P<0.01$, $\left.* * * P<0.001\right)$. DNA-PK, DNA-PKcs.

therefore, we also tested whether FASN causes resistance to DNA-PK inhibition in the presence of DNA damage. As shown in Supplemental Fig. S3, no change occurred in the cellular response to NU7441 in the presence of doxorubicin at its $\mathrm{IC}_{20}$ concentration. No significant difference in cellular response to CC-115 was seen in the presence of doxorubicin at its $\mathrm{IC}_{20}$ concentration, although there may be a trend toward resistance (Supplemental Fig. S3).

Because CC-115 is a dual mTOR/DNA-PK inhibitor, we next tested whether its inhibition of the mTOR pathway was impaired in the drug-resistant M3K cells. As shown in Fig. 1E, CC-115 inhibition of constitutive activation of S6K and phosphorylation of S6, downstream targets of mTOR, were significantly impaired in M3K cells compared with MCF7 cells. Based on these findings, we conclude that FASN does not play a role in DNA-PK inhibitor resistance.

CC-115 Accumulation Is Reduced in Drug-Resistant M3K Cells. To investigate further the molecular mechanism of CC-115 resistance, we tested the possible involvement of ABCG2, which is also overexpressed in M3K cells (Liu et al., 2005) (see also Fig. 2A), by first determining the accumulation of CC-115 in M3K versus MCF7 cells. As shown in
Supplemental Fig. S4, CC-115 is autofluorescent with an emission wavelength at $421 \mathrm{~nm}$, which was used to monitor its intracellular accumulation. As shown in Fig. 2, B and C, CC115 accumulation was much lower in M3K cells, with an $\sim 80 \%$ reduction compared with MCF7 cells.

Sensitization of M3K Cells to CC-115 by Inhibiting ABCG2. To determine whether ABCG2 is responsible for the reduced CC-115 accumulation in M3K cells, we tested the effect of ABCG2-specific inhibitors C8 and FTC (Rabindran et al., 2000; Peng et al., 2009) on CC-115 accumulation in M3K cells. As shown in Fig. 3, A and B, both C8 and FTC dose dependently increased CC-115 accumulation in M3K cells. Both C8 and FTC also significantly reversed CC-115 resistance of M3K cells (Fig. 3, C and D). Furthermore, inhibiting ABCG2 with C8-sensitized M3K cells to CC-115 inhibition of mTOR activation of S6K (Fig. 3E).

ABCG2 Overexpression Reduces CC-115 Accumulation in Both HEK293 and MCF7 Cells. To determine further the role of ABCG2 in CC-115 efflux and resistance, we took advantage of human embryonic kidney cell line 293 and MCF7 cells stably transfected with ABCG2 (HEK293/ABCG2venus, which contains a venus tag and MCF7/ABCG2, which 

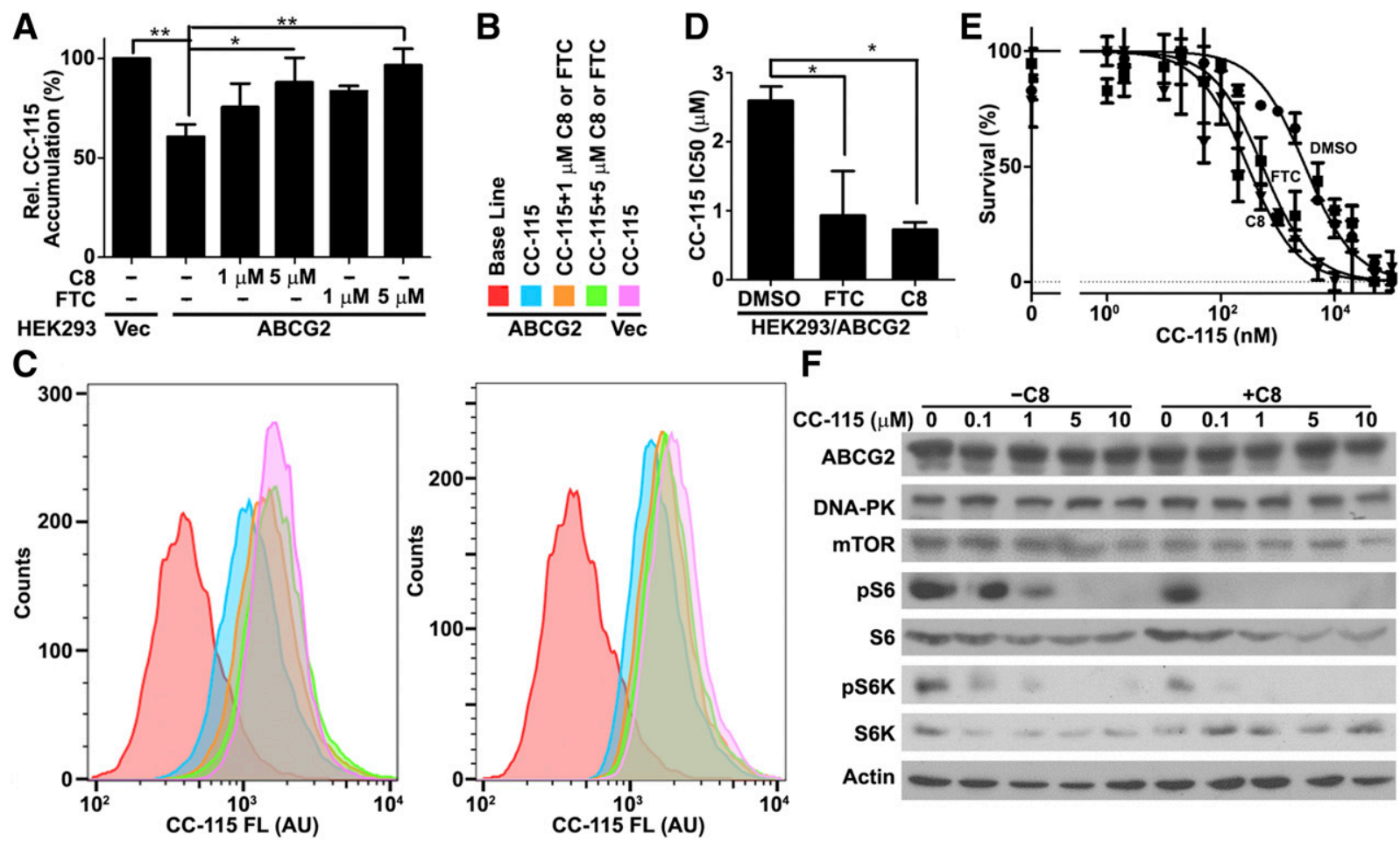

Fig. 5. Inhibition of ABCG2 and reversal of CC-115 resistance. (A-C) CC-115 accumulation in HEK293/Vec versus HEK293/ABCG2-venus cells in the absence or presence of ABCG2 inhibitors C8 and FTC as determined using FACS analysis. (D and E) CC-115 IC 50 in and representative dose-dependent survival curves of HEK293/ABCG2-venus cells in the absence and presence of inhibitor C8 or FTC as determined using methylene blue assay. (F) CC-115 inhibition of mTOR activation in HEK293/ABCG2-venus cells in the absence or presence of ABCG2 inhibitor C8 as determined by monitoring phosphorylation of S6 and S6K using Western blot analysis. ANOVA was used for statistical analysis of CC-115 accumulation $(\mathrm{A})$ and $\mathrm{IC}_{50}(\mathrm{C})(n=3$ to 4 ; $\left.{ }^{*} P<0.05,{ }^{* *} P<0.01,{ }^{* * *} P<0.001\right)$. DNA-PK, DNA-PKes.

does not), and their respective control cells transfected with vector (HEK293/Vec, venus tagged, and MCF7/Vec) (see Fig. 4A). Using these cells, we first determined CC-115 accumulation as described already. As shown in Fig. 4, B and C, accumulation of CC-115 in HEK293/ABCG2-venus and MCF7/ABCG2 cells was significantly reduced compared with their respective control cells. We next determined their response to CC-115 in a survival assay. As shown in Fig. 4,
$\mathrm{D}$ and $\mathrm{E}$, the $\mathrm{CC}-115 \mathrm{IC}_{50}$ was significantly increased in both HEK293/ABCG2-venus and MCF7/ABCG2 compared with their respective control cells. The CC-115 inhibition of the mTOR activation of S6K, as indicated by the level of phosphorylated S6K and S6, was also reduced by ABCG2 overexpression in these cells (Fig. $4 \mathrm{~F}$ ). We also tested the effect of CC-115 on mTORC2 by determining its effect on AKT phosphorylation. As shown in Supplemental Fig. S5A, CC-115

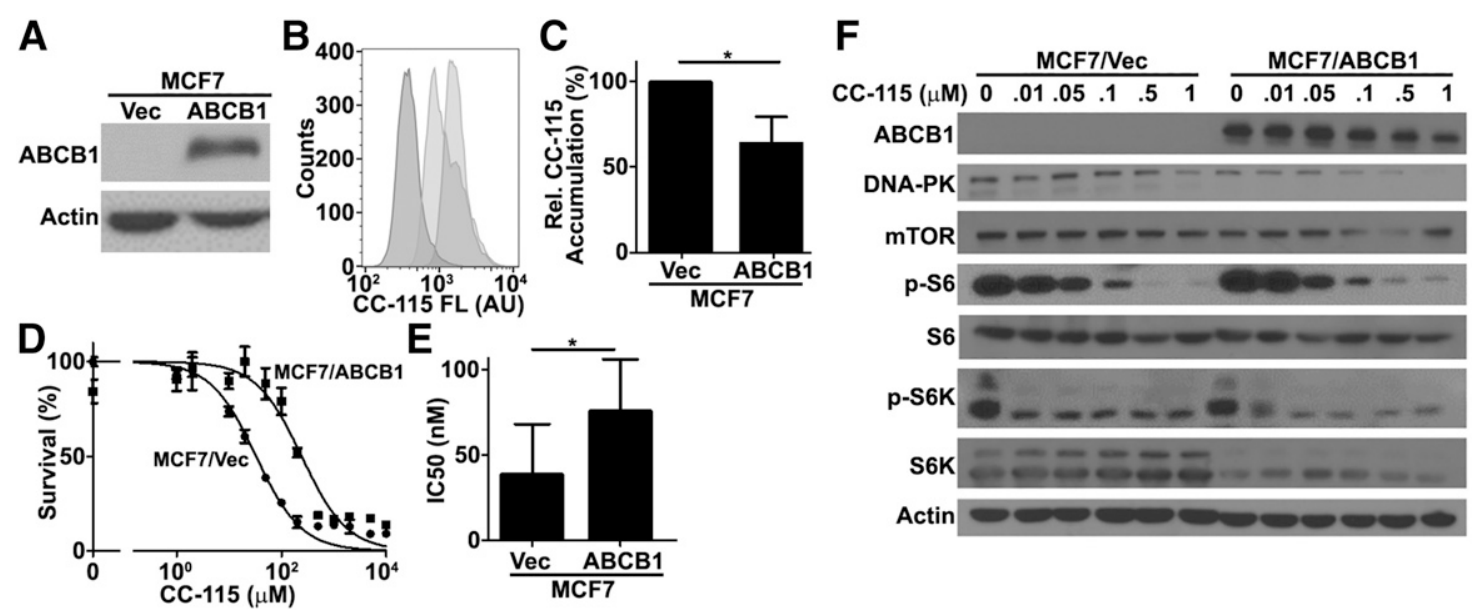

Fig. 6. CC-115 is a substrate for ABCB1. (A) Western blot analysis of ABCB1 expression in MCF7/ABCB1 and the control MCF7/Vec cells. (B and C) CC115 accumulation in MCF7/Vec and MCF7/ABCB1 cells as determined using FACS analysis. (D and E) CC-115 IC 50 in and representative survival curves of MCF7/ABCB1 and the control MCF7/Vec cells. (F) Western blot analysis of ABCB1 effect on CC-115 inhibition of mTOR signaling in MCF7/Vec and MCF7/ABCG2 cells. Note that p85 was also detected using S6K and p-S6K antibodies. The $t$ test was used for statistical analysis of CC-115 accumulation $(\mathrm{C})$ and $\mathrm{IC}_{50}(\mathrm{E})(n=3 ; * P<0.05$, **P $<0.01$, ***P $<0.001)$. 
inhibited AKT phosphorylation, and ABCG2 overexpression reduced CC-115 inhibition of AKT phosphorylation. Thus, CC115 likely inhibits both mTORC1 and mTORC2, which can be reduced by $\mathrm{ABCG} 2$ expression.

To validate the role of ABCG2 in CC-115 resistance and accumulation, we determined whether inhibiting the function of ABCG2 could reverse the ectopic ABCG2-induced resistance and accumulation reduction of CC-115. As shown in Fig. 5, A-C, both C8 and FTC significantly reversed ABCG2induced CC-115 accumulation reduction. Moreover, both C8 and FTC significantly reduced CC-115 $\mathrm{IC}_{50}$ in HEK293/ ABCG2-venus cells (Fig. 5, D and E). Consistently, C8 was able to sensitize HEK293/ABCG2-venus cells to CC-115 inhibition of mTORC1 pathway, as indicated by S6K and S6 phosphorylation (Fig. 5F), and mTORC2, as indicated by AKT phosphorylation (Supplemental Fig. S5B).

Role of ABCB1 in CC-115 Resistance. It is known that $\mathrm{ABC}$ transporters involved in drug resistance have overlapping substrates; thus, we next tested whether other $A B C$ transporters, such as ABCB1, may also contribute to CC-115 resistance. For this purpose, we took advantage of MCF7 cells that overexpress ectopic ABCB1 (BC19 cells) and tested CC115 accumulation and resistance compared with vectortransfected MCF7 cells (MCF7/Vec) (Fig. 6A). As shown in Fig. 6, B and C, CC-115 accumulation is significantly reduced in BC19 compared with the control MCF7/Vec cells. The $\mathrm{IC}_{50}$ of CC-115 was also significantly increased in BC19 cells compared with the MCF7/Vec cells (Fig. 6, D and E). When cells were treated with increasing concentrations of CC-115, there was a slight change in S6K and S6 phosphorylation compared with MCF7/Vec cells (Fig. 6F). Thus, ABCB1, in addition to $\mathrm{ABCG} 2$, may also contribute to $\mathrm{CC}-115$ resistance by reducing its intracellular accumulation.

\section{Discussion}

In this study, we showed that ABCG2 expression plays a major role in $\mathrm{CC}-115$ resistance by reducing its intracellular accumulation and its inhibition of mTOR pathway. ABCB1, to a lesser extent, may also contribute to CC-115 resistance via a similar mechanism. Clearly, CC-115 may be a substrate of multiple $\mathrm{ABC}$ transporters and reduction in clinical response is expected in patients that express any of these $\mathrm{ABC}$ transporters.

Based on these findings, it is tempting to speculate that ABCG2 and ABCB1 may be used as markers to stratify patients into treatment groups for future clinical trials. The observed variability in CLL patient response in a past trial may be due to different expression of ABCG2 or ABCB1. Previously, it has been shown that ABCG2 and ABCB1 are expressed in leukemia patients, and their expression influences the outcome of these patients, who were subjected to chemotherapy (Svirnovski et al., 2009; Mo and Zhang, 2012). Currently, there is an ongoing clinical trial of CC-115 for glioblastoma; however, it is also well known that ABCG2 and ABCB1 are both highly expressed in the blood-brain barrier (Loscher and Potschka, 2005), which may affect the outcome of this trial.

It is noteworthy that the resistance of M3K cells to CC-115, compared with the parental MCF7 cells, is $\sim 50$-fold, whereas the resistance of MCF7/ABCG2 cells to CC- 115 compared with MCF7/Vec cells is only $\sim 2$-fold. This discrepancy may be due to the difference in the expression level of ABCG2 (see Supplemental Fig. S6). This is also supported by the fact that ABCG2 overexpression in HEK293 cells, which also express a much higher level of ABCG2 than MCF7/ABCG2 cells (Supplemental Fig. S6), induced a much larger ( 10 fold) CC-115 resistance compared with HEK293/Vec cells. It is also possible that ABCG2 in M3K cells has a gain of function in increased activity of transporting CC-115 owing to its mutation of $\mathrm{Arg}^{482}$ to $\mathrm{Thr}^{482}$ (Alqawi et al., 2004). Lastly, M3K cells are known to harbor other mechanisms of drug resistance (Liu et al., 2005, 2006, 2008; Wu et al., 2016a) that may also contribute to $\mathrm{CC}-115$ resistance in $\mathrm{M} 3 \mathrm{~K}$ cells.

It is also noteworthy that although ABCB1 expression causes resistance to CC-115 and reduces intracellular accumulation of CC-115 and CC-115 inhibition of mTOR pathway, these effects appear to be less than that of ABCG2. The reason for this difference is unknown; however, it is possible that CC115 has a higher affinity to ABCG2 than to ABCB1. It is also possible that the expression level of $A B C B 1$ is less than that of ABCG2 as discussed already for ABCG2 in different expressing host cells. These possibilities remain to be tested in future studies.

Although FASN upregulates DNA-PK activity (Wu et al., 2016a), FASN overexpression does not appear to contribute to cellular resistance to the DNA-PK inhibitory activity of CC-115 or a DNA-PK-selective inhibitor, NU7441. Interestingly, we have shown that FASN overexpression causes resistance to DNA-damaging drugs (Liu et al., 2013) and inhibitors of PARP1 (submitted manuscript), a mediator of FASN-induced up-regulation of DNA-PK activity (Wu et al., 2016a). Although it remains to be determined, the failure of FASN overexpression in causing resistance to DNA-PK inhibition may be due to the fact that FASN overexpression leads only to an increase in activity and not in expression of DNA-PK, which may be insufficient to overcome the effect of DNA-PK inhibition.

\section{Acknowledgments}

We thank Dr. Susan Bates for MCF7/AdVp3000 cells and Dr. Julie Horton for BC19 cells.

\section{Authorship Contributions}

Participated in research design: Beebe, Zhang.

Conducted experiments: Beebe.

Performed data analysis: Beebe, Zhang.

Wrote or contributed to the writing of the manuscript: Beebe, Zhang.

\section{References}

Alqawi O, Bates S, and Georges E (2004) Arginine482 to threonine mutation in the breast cancer resistance protein ABCG2 inhibits rhodamine 123 transport while increasing binding. Biochem $J$ 382:711-716.

Hammel M, Yu Y, Mahaney BL, Cai B, Ye R, Phipps BM, Rambo RP, Hura GL, Pelikan M, So S, et al. (2010) Ku and DNA-dependent protein kinase dynamic conformations and assembly regulate DNA binding and the initial non-homologous end joining complex. J Biol Chem 285:1414-1423.

Horton JK, Vanoye CG, and Reuss L (1998) Swelling-activated chloride currents in a drug-sensitive cell line and a $\mathrm{P}$ glycoprotein-expressing derivative are underlied by channels with the same pharmacological properties. Cell Physiol Biochem 8: $246-260$.

Hung CM, Garcia-Haro L, Sparks CA, and Guertin DA (2012) mTOR-dependent cell survival mechanisms. Cold Spring Harb Perspect Biol 4:a008771.

Liu H, Liu Y, and Zhang JT (2008) A new mechanism of drug resistance in breast cancer cells: fatty acid synthase overexpression-mediated palmitate overproduction. Mol Cancer Ther 7:263-270.

Liu H, Wu X, Dong Z, Luo Z, Zhao Z, Xu Y, and Zhang JT (2013) Fatty acid synthase causes drug resistance by inhibiting $\mathrm{TNF}-\alpha$ and ceramide production. $J$ Lipid Res 54:776-785. 
Liu Y, Liu H, Han B, and Zhang JT (2006) Identification of 14-3-3sigma as a contributor to drug resistance in human breast cancer cells using functional proteomic analysis. Cancer Res 66:3248-3255.

Liu Y, Peng H, and Zhang JT (2005) Expression profiling of ABC transporters in a drug-resistant breast cancer cell line using AmpArray. Mol Pharmacol 68: $430-438$

Löscher W and Potschka H (2005) Blood-brain barrier active efflux transporters: ATP-binding cassette gene family. NeuroRx 2:86-98.

Lovejoy CA and Cortez D (2009) Common mechanisms of PIKK regulation. DNA Repair (Amst) 8:1004-1008.

Mo W and Zhang JT (2012) Human ABCG2: structure, function, and its role in multidrug resistance. Int $J$ Biochem $\mathrm{Mol}$ Biol 3:1-27.

Mortensen DS, Perrin-Ninkovic SM, Shevlin G, Elsner J, Zhao J, Whitefield B, Tehrani L, Sapienza J, Riggs JR, Parnes JS, et al. (2015) Optimization of a series of triazole containing mammalian target of rapamycin (mTOR) kinase inhibitors and the discovery of CC-115. J Med Chem 58:5599-5608.

Munster PN, Mahipal A, Nemunaitis JJ, Mita MM, Paz-Ares LG, Massard C, Mikkelsen T, Cruz C, Rathkopf DE, Blumenschein GR, et al. (2016) Phase I trial of a dual TOR kinase and DNA-PK inhibitor (CC-115) in advanced solid and hematologic cancers. J Clin Oncol 34 (15 Suppl):2505.

Oliver MH, Harrison NK, Bishop JE, Cole PJ, and Laurent GJ (1989) A rapid and convenient assay for counting cells cultured in microwell plates: application for assessment of growth factors. J Cell Sci 92:513-518.

Peng H, Dong Z, Qi J, Yang Y, Liu Y, Li Z, Xu J, and Zhang JT (2009) A novel two mode-acting inhibitor of ABCG2-mediated multidrug transport and resistance in cancer chemotherapy. PLoS One 4:e5676.

Rabindran SK, Ross DD, Doyle LA, Yang W, and Greenberger LM (2000) Fumitremorgin $\mathrm{C}$ reverses multidrug resistance in cells transfected with the breast cancer resistance protein. Cancer Res 60:47-50.

Schinkel AH and Jonker JW (2003) Mammalian drug efflux transporters of the ATP binding cassette (ABC) family: an overview. Adv Drug Deliv Rev 55: $3-29$.
Svirnovski AI, Shman TV, Serhiyenka TF, Savitski VP, Smolnikova VV, and Fedasenka UU (2009) ABCB1 and ABCG2 proteins, their functional activity and gene expression in concert with drug sensitivity of leukemia cells. Hematology 14:204-212.

Thijssen R, Ter Burg J, Garrick B, van Bochove GG, Brown JR, Fernandes SM, Rodríguez MS, Michot JM, Hallek M, Eichhorst B, et al. (2016) Dual TORK/DNAPK inhibition blocks critical signaling pathways in chronic lymphocytic leukemia. Blood 128:574-583.

Tsuji T, Sapinoso LM, Tran T, Gaffney B, Wong L, Sankar S, Raymon HK, Mortensen DS, and Xu S (2017) CC-115, a dual inhibitor of mTOR kinase and DNA-PK, blocks DNA damage repair pathways and selectively inhibits ATM-deficient cell growth in vitro. Oncotarget 8:74688-74702.

Wu X, Dong Z, Wang CJ, Barlow LJ, Fako V, Serrano MA, Zou Y, Liu JY, and Zhang JT (2016a) FASN regulates cellular response to genotoxic treatments by increasing PARP-1 expression and DNA repair activity via NF-кB and SP1. Proc Natl Acad Sci USA 113:E6965-E6973.

Wu X, Dong Z, Wang CJ, Barlow LJ, Fako V, Serrano MA, Zou Y, Liu JY, and Zhang JT (2016b) FASN regulates cellular response to genotoxic treatments by increasing PARP-1 expression and DNA repair activity via NF-кB and SP1. Proc Natl Acad Sci U S A 113:E6965-E6973.

Xu J, Liu Y, Yang Y, Bates S, and Zhang JT (2004) Characterization of oligomeric human half-ABC transporter ATP-binding cassette G2. J Biol Chem 279:19781-19789.

Xu J, Peng H, Chen Q, Liu Y, Dong Z, and Zhang JT (2007) Oligomerization domain of the multidrug resistance-associated transporter ABCG2 and its dominant inhibitory activity. Cancer Res 67:4373-4381.

Address correspondence to: Jian-Ting Zhang, Department of Cancer Biology, University of Toledo College of Medicine and Life Sciences, 3000 Arlington Ave., MS1010, Room 413, Toledo, OH 43614. E-mail: jianting.zhang@ utoledo.edu 